\title{
Orbital alignment effect in a supersonic jet: spin-changing collisions in the $\mathrm{Ca}\left(4 \mathrm{~s} 5 \mathrm{p}^{1} \mathrm{P}_{1}\right)+\mathrm{Ar}$ system
}

\author{
Yih-Shing Duh, King-Chuen Lin \\ Department of Chemistry, National Taiwan University, Taipei, Taiwan, ROC \\ and Institute of Atomic and Molecular Sciences, Academia Sinica, P.O. Box 23-166, Taipei 10764, Taiwan, ROC
}

and

Wei-Tzou Luh

Institute of Atomic and Molecular Sciences, Academia Sinica, P.O. Box 23-166, Taipei 10764, Taiwan, ROC

Received 19 May 1992

\begin{abstract}
We have studied orbital alignment effects in a single supersonic jet. The Ar gas behind the nozzle is used as both the collision partner and the carrier gas for free jet expansion. The Ca $4 s_{\mathrm{p}}{ }^{1} \mathrm{P}$, state is prepared by a linearly polarized laser. By varying the angle of the laser polarization with respect to the relative velocity vector of the $\mathrm{Ca}$ and $\mathrm{Ar}$ collision, either the asymptotic $\Sigma$ or $\Pi$ molecular state related to the $\mathrm{Ca}-\mathrm{Ar}$ collision can be prepared and followed. The measurement of polarization upon the $\mathrm{Ca}\left(4 \mathrm{~s} 5 \mathrm{p}{ }^{1} \mathrm{P}_{1} \rightarrow 4 \mathrm{~s} 5 \mathrm{p}{ }^{3} \mathrm{P}_{J}\right.$ ) spin-changing collision by Ar gives a value of $19 \% \pm 1.5 \%$ at a background pressure of about $55 \mathrm{mTorr}$. The result is in excellent agreement with the crossed-beam value $21 \% \pm 7 \%$ of Leone and co-workers.
\end{abstract}

\section{Introduction}

Orbital alignment effects of an electronically excited metal atom on the cross sections of chemical reactions or energy transfer processes have drawn much attention from experimentalists [1-7] and theoreticians [8-10] during the last decade. Investigations have provided some insights into the effect of the repulsive and attractive characters of a collision trajectory in near resonant energy transfer [3,4,7-12], electron-transfer reactions [13], chargeexchange processes $[14,15]$, association ionization reactions $[16,17]$, quenching processes $[6,18]$, or fine-structure mixing collisions $[19,20]$.

A crossed-beam apparatus has been mostly employed for the studies of atomic orbital alignment. For instance, the alignment dependence of energy transfer in the Ca-rare-gas spin-changing collision

Correspondence to: K.-C. Lin, Institute of Atomic and Molecular Sciences, Academia Sinica, P.O. Box 23-166, Taipei 10764, Taiwan, ROC. has been carried out by Leone and co-workers using a crossed-beam configuration [3-5,7], consisting of an effusive beam for the metal atoms and a supersonic jet for the collision partner. The Ca $5{ }^{1} \mathrm{P}_{1}$ state has been first prepared by a linearly polarized laser. The fluorescence intensity of the $\mathrm{Ca} 5{ }^{3} \mathrm{P}_{J} \rightarrow 3^{3} \mathrm{D}_{J}$ transition at $617 \mathrm{~nm}$ was then monitored as the angle $\boldsymbol{\theta}$ of the incident electric field vector with respect to the relative velocity vector was varied. They have demonstrated that the measured fluorescence intensity $I$ essentially follows a $\cos 2 \theta$ dependence, and the signal appears to be the maximum when the $p$ orbital is aligned perpendicular to the relative velocity vector, and to be the minimum when aligned parallel. The maximum intensity $I_{\max }$ or the minimum intensity $I_{\min }$ are related to the cross section of a near-resonant energy transfer in which the asymptotic $\Pi$ or $\Sigma$ molecular state are locked and followed, respectively. Accordingly, the corresponding polarization can be defined as $P=\left(I_{\max }-I_{\min }\right) /$ $\left(I_{\max }+I_{\min }\right)$. For the case of $\mathrm{Ca}-\mathrm{Ar}$, the polarization has a value of $21 \% \pm 7 \%$ [7]. 
The second method is by the far-wing scattering technique, with which Kleiber and co-workers have studied the orbital alignment dependence of the $\mathrm{Ca}\left(5^{1} \mathrm{P}_{1} \rightarrow 5^{3} \mathrm{P}_{J}\right)$ energy transfer process by collision with $\mathrm{He}[21,22]$. In the experiment a laser is tuned into the collision-broadened wings of the $\mathrm{Ca} 4{ }^{1} \mathrm{~S}_{0} \rightarrow 5{ }^{1} \mathrm{P}_{1}$ transition, exciting the $\mathrm{CaHe}$ quasimolecule at long range. The absorption in the far red wing is expected to predominantly excite the attractive $\Pi$ state of $\mathrm{CaHe}$, while the absorption in the far bluc wing corresponds to excitation of the repulsive $\Sigma$ state $[21,22]$. The observed cross section dependence of the red/blue wing detunings can be characterized by a spin-orbit curve crossing model. Conceptually, if the long-range nonadiabatic $\Pi-\Sigma$ electronic mixing is negligible, then the alignment effects obtained by the far-wing scattering technique are comparable with that by the crossed-beam methods. However, it has been found that the $\Sigma$ and $\Pi$ symmetry are possibly scrambled when the colliders are approaching each other $[2-4,9]$. That is, the orbital initially aligned in the crossed-beam cannot be preserved completely in the collision. Kleiber et al. have pointed out that the extent of the orbital scrambling can be reduced when the orbital alignment is prepared at a shorter internuclear distance $[21,22]$. The alignment effect would reach almost $100 \%$ as a result of $\geqslant 70 \mathrm{~cm}^{-1}$ detuning from the atomic transition [22]. However, this technique may suffer from an extremely weak scattering signal for further detuning. In addition, the $\Pi$ or $\Sigma$ molecular states should be presumed to represent an entirely attractive or repulsive curve, respectively. The detunings may otherwise lead to a confusion of orbital admixtures.

The third method for the orbital alignment measurement is by a half-collision study of a van der Waals complex formed between the metal and the rare gas [23-26]. The $\Sigma$ (or $\Pi$ ) molecular state of the complex will be excited selectively as a result of blue-shift (or red-shift) near the atomic transition. By observing the "action spectra" of the dissociated metal atom with a probe laser fixed at a particular atomic transition, insight into the alignment effect on the chemical reaction or energy transfer can also be gained. Soep, Breckenridge and co-workers have successfully conducted half-collision studies for $\mathrm{CdXe}$ [27], $\mathrm{ZnXe}$ [28], and HgR [29] ( $\mathrm{R}=$ rare gas or small molecules) van der Waals complexes.

Alternative to the above crossed-beam technique, we have performed our study on the alignment effect in a single supersonic jet. In order for a comparison with the crossed-beam measurement, the spinchanging collision in the $\mathrm{Ca}-\operatorname{Ar}\left(4 s 5 \mathrm{p}{ }^{1} \mathrm{P}_{1}-4 s 5 p{ }^{3} \mathrm{P}_{J}\right)$ system is selected as an example. In our apparatus the $\mathrm{Ca}$ metal is seeded in a supersonic free jet, where the Ar gas behind the nozzle acts as both the collision partner and the carrier gas for free expansion. A linearly polarized laser is used as the excitation source. With a double Fresnel rhomb rotator, the electric field vector of impinging laser can be rotated with respect to the molecular-beam direction. Analogous to the crossed-beam configuration, the perpendicular alignment corresponds to excitation to the asymptotic $\Pi$ molecular state, while the parallel alignment can result in excitation of the asymptotic $\Sigma$ state. The experiment is done under the condition that the atoms entrained in the beam encounter only a single collision at the interaction region during the period of fluorescence observation. The obtained alignment effect is quite consistent with that reported by Leone and co-workers in the crossed-beam studies [7].

\section{Experimental}

The apparatus shown in fig. 1 contained a supersonic free jet, in which the $\mathrm{Ca}$ metal was stored in a $3 \mathrm{~cm} \times 6 \mathrm{~cm}$ (diameter $\times$ length) cylinder nozzle with an orifice of $2 \mathrm{~mm}$ diameter. The nozzle orifice was mounted with a pair of collimating pinholes of $2 \mathrm{~mm}$ diameter to make the $\mathrm{Ca}$ beam have a better directionality. The spacing between the two pinholes was also $2 \mathrm{~mm}$. The collision partner Ar gas was used as the carrier gas of the Ca nozzle; the stagnation pressure was maintained at at most 1 atm throughout the work, and the background pressure was regulated in the range of $10^{-3}-8$ Torr and monitored with an MKS Baratron gauge.

The resulting supersonic jet from the Ca nozzle at $800 \pm 2 \mathrm{~K}$ has a very narrow speed distribution and good directionality. Both the $\mathrm{Ca}$ atom and its collision partner Ar entrained in the beam have parallel velocities in the free-collision zone. Although the collisions cannot be ideally free, their relative veloc- 


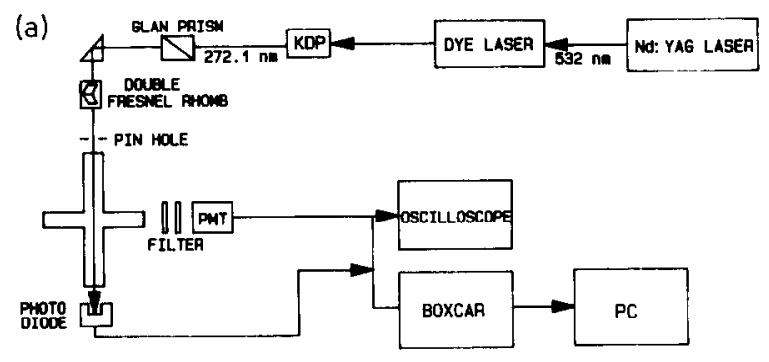

(b)

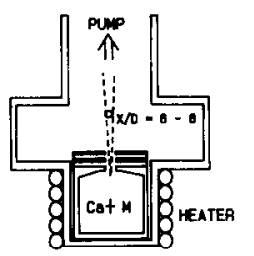

Fig. 1. Schematics of (a) the experimental apparatus and (b) the nozzle design.

ity in the center-of-mass frame (or collision axis) is along the free jet direction. In section 3 we will discuss in detail the collision frequency encountered in the interaction zone between the jet and the excitation laser beam.

The construction of a six-armed stainless steel chamber was similar to that of the heat-pipe oven used for the $\mathrm{K}^{*}+\mathrm{H}_{2}$ and $\mathrm{Mg}^{*}+\mathrm{H}_{2}$ experiments $[30,31]$, except that a Ca nozzle was put tightly into the bottom arm. A pair of semi-cylindrical heaters were wrapped around the arm. The nozzle temperature was maintained at $800 \pm 2 \mathrm{~K}$ and monitored by a thermocouple.

A dye laser, operated with the dye Fluorescein 548, was pumped by the second harmonic of an Nd: YAG laser. Its output frequency was then doubled with a $\mathrm{KDP}$ crystal and tuned to the $\mathrm{Ca} 4{ }^{1} \mathrm{~S}_{0} \rightarrow 5^{1} \mathrm{P}_{1}$ transition at $272.1 \mathrm{~nm}$. The linearly polarized radiation was refined with a Glan prism polarizer and the orientation of its electric field vector could then be rotated with a double Fresnel rhomb polarization rotator. The unfocused radiation was collimated to a beam size of $3 \mathrm{~mm}$ diameter through a pinhole. The optical alignment was optimized such that the power and the direction of incident radiation monitored behind the chamber were independent of the electric field orientation. The laser energy was maintained in the range of $200-300 \mu \mathrm{J}$ to avoid multiphoton effects. The laser interaction region with the super- sonic jet was $14 \pm 2 \mathrm{~mm}$ above the nozzle orifice. The parallel alignment of the electric field vector with respect to the collision axis corresponds to excitation of the asymptotic $\Sigma$ molecular state, while the perpendicular alignment will result in excitation of the asymptotic $\Pi$ state.

The collision-induced fluorescence (CIF) signal due to the transition $5{ }^{3} \mathrm{P}_{J} \rightarrow 3{ }^{3} \mathrm{D}_{J}$ at $617 \mathrm{~nm}$ was measured as a function of the rotation angle of the laser polarization. The CIF was detected by a photomultiplier tube, which was positioned at $90^{\circ}$ to the incident laser beam. The appropriate filters were used for reducing the scattering light. The signal was then processed by a boxcar averager and stored in a microcomputer for further data analysis.

\section{Results and discussion}

Fig. 2 shows the Ca atomic fluorescence signal for

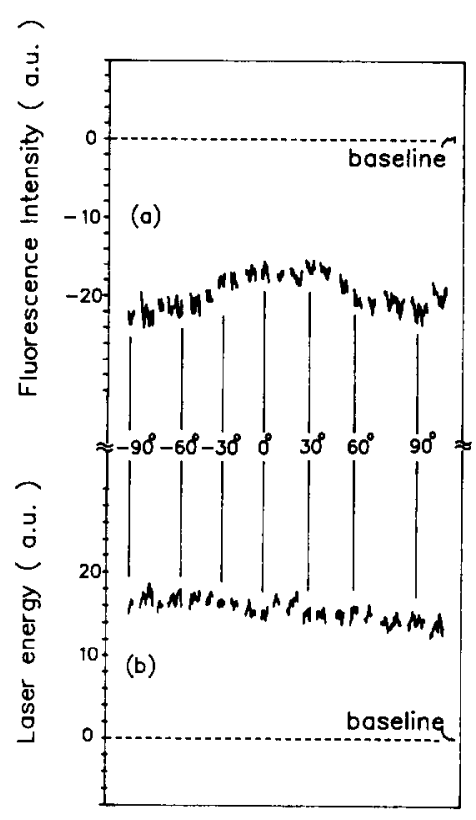

Fig. 2. The raw experimental data as a function of rotating the laser polarization angle with respect to the supersonic jet direction: (a) fluorescence intensity of $\mathrm{Ca}^{3} \mathrm{P}_{J} \rightarrow 3^{3} \mathrm{D}_{J}$ transition and (b) laser energy as a function of the alignment of the laser polarization. Note that slight decay of the energy results from a long period of data acquisition, but not caused by the alignment factor. The intensity around $-90^{\circ}$ is smaller than that around $90^{\circ}$ duc to a slight decay of the laser power. 
the $5{ }^{3} \mathrm{P}_{\mathrm{J}} \rightarrow 3{ }^{3} \mathrm{D}_{\mathrm{J}}$ transition at $617 \mathrm{~nm}$ as a function of the angle of the electric field vector with respect to the jet beam direction. After normalization to the laser energy, the fluorescence intensity is found to vary as the direction of the polarized radiation changes. The fluorescence intensity is the largest as the electric field vector is in a perpendicular alignment, and the smallest in a parallel alignment.

Note the experiment is under the condition that the stagnation pressure $P_{0}$ behind the nozzle reaches at most $1 \mathrm{~atm}$ and the background pressure $P_{\mathrm{b}}$ of the chamber is pumped below $100 \mathrm{mTorr}$. The distance at which the Mach disk occurs is roughly estimated to be $120 \mathrm{~mm}$ downstream from the nozzle orifice by [32]

$X=0.67 D\left(P_{0} / P_{\mathrm{b}}\right)$,

where $D=2 \mathrm{~mm}$ is the nozzle diameter. The interaction region with the radiation, located $14 \pm 2 \mathrm{~mm}$ downstream of the orifice, is considered to be in the free expansion zone. Many-body collisions in the beam can only be significant within the $X / D \leqslant 5$ region [33]. Therefore, the interaction zone lies in the molecular flow region, where bimolecular collisions will dominate if collisions cannot be ideally free [33]. It becomes obvious that the asymptotic $\Pi$ molecular state (or the asymptotic $\Sigma$ molecular state) of the Ca-Ar collision can be aligned initially when the electric field vector is rotated to be perpendicular (or parallel) to the $\mathrm{Ca}$ beam direction.

Using the following equation we can estimate the bimolecular collision rate occurring in the molecular flow region for the supersonic free jet [32]:

$z=2^{1 / 2} n_{0} \sigma v_{0}\left[1+\frac{1}{2}(\gamma-1) M_{\mathrm{eff}}^{2}\right]^{-(1 / 2)(\gamma+1) /(\gamma-1)}$.

Here the reservoir density $n_{0}$ gives $9.2 \times 10^{18}$ parti$\mathrm{cle} / \mathrm{cm}^{3}$ for 1 atm nozzle pressure; the collision cross section $\sigma$ is considered to be $10 \AA^{2}$ assuming a model of hard-sphere collisions; the mean velocity $v_{0}$ in the reservoir gives $6 \times 10^{4} \mathrm{~cm} / \mathrm{s}$ at the nozzle temperature $800 \mathrm{~K} . \gamma$ indicates the heat capacity ratio $C_{p} / C_{V}$. The effective Mach number downstream in the interaction region can be estimated to be 12 by [32]

$$
\begin{gathered}
M_{\text {eff }}=3.26(X / D-0.075)^{0.67} \\
-0.61(X / D-0.075)^{-0.67,}
\end{gathered}
$$

where $X / D=7$ is used in the experiment. With the above parameters substituted into eq. (2), we obtain the collision rate approximately to be $6 \times 10^{5}$ collision/s. This implics that a sccondary collision in the interaction region may well be negligible during the $10-100$ ns period for the $\mathrm{Ca}\left(5^{3} \mathrm{P}_{J}-3{ }^{3} \mathrm{D}_{J}\right)$ fluorescence observation. The argument is still valid, even though the collision cross section is enlarged by 10 times.

The polarization is defined as $\left(I_{\max }-I_{\min }\right) /$ $\left(I_{\max }+I_{\min }\right)$, in which $I_{\max }$ and $I_{\min }$ are related to the cross sections of energy transfer. They can be obtained by measurement of the fluorescence from the $5^{3} \mathrm{P}_{1}$ state, as the excited Ca p orbital is aligned perpendicular or parallel, respectively, to the collision axis. The polarization obtained in this work is found to be dependent upon the background pressure. As shown in fig. 3, the alignment effect (or polarization) diminishes as the background Ar pressure increases. The measured polarization (in fig. 4) shows an exponential-like dependence on the Ar pressure, and the alignment effect is finally depleted when the Ar background pressure is higher than 5 Torr. The decaying dependence is closely related to both the quenching rate of the $\mathrm{Ca} 5^{1} \mathrm{P} \rightarrow 5^{3} \mathrm{P}$ transition and the depolarization effect. The orbital alignment effect for the spin-changing collision of the Ca-Ar system under 55 mTorr background pressure approaches $19 \% \pm 1.5 \%$. The result. The result is found

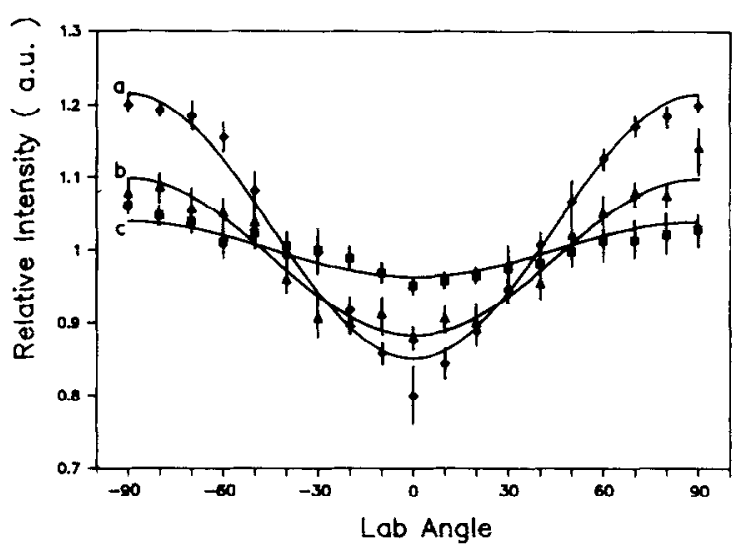

Fig. 3. Fluorescence intensity of the $5{ }^{3} \mathrm{P}_{J} \rightarrow 3^{3} \mathrm{D}_{J}$ transition as a function of the laser polarization angle at various chamber background pressures of Ar: (a) $68 \mathrm{mT}$ Trr, (b) $280 \mathrm{mTorr}$, and (c) 1.4 Torr. 




Fig. 4. Background Ar pressure dependence of the polarization on the $\mathrm{Ca} 5{ }^{1} \mathrm{P}_{1} \rightarrow 5^{3} \mathrm{P}_{J}$ spin-changing collision.

to be very consistent with the value of $21 \% \pm 7 \%$ obtained by Leone and co-workers using the crossedbeam apparatus [7]. A larger value can be expected if extrapolation will be extended to the zero pressure, at which the polarization can be considered to be free of the interference from quenching or depolarization processes. The latter occurs when the spatial orientation of angular momentum of the system changes during the collision. In work still underway, we have also found that the polarization of $\mathrm{Ca}-\mathrm{H}_{2}$ reaches $12 \%$ below 66 mTorr background pressure [34], consistent with the value of $13 \% \pm 1 \%$ reported previously [35]. A preliminary result on $\mathrm{Mg}+\mathrm{H}_{2}$ reaction, carried out using a similar device, shows that the rotational quantum state distribution of $\mathrm{MgH}$ product depends upon the alignment of the polarizcd radiation [36]. The facts described above demonstrate that it is possible to conduct orbital alignment experiments on both collisional energy transfer and chemical reaction processes with a single supersonic jet.

Analogous to our apparatus, an $\mathrm{Na}$ effusive oven has been employed in the study of the associative ionization reaction of $\mathrm{Na}\left(3^{2} \mathrm{P}_{3 / 2}\right)+\left(\mathrm{Na}\left(3^{2} \mathrm{P}_{3 / 2}\right)\right.$ $\rightarrow \mathrm{Na}_{2}^{+}+\mathrm{e}[16,17]$. The excited $\mathrm{Na}$ atoms collide because there is a distribution of speeds within the atomic beam. The relative velocity appears to be parallel to the beam $[16,17]$. Although the design of the single-beam configuration is similar, the beam characteristics between the effusive oven and the supersonic jet are quite different. First of all, the density of an effusive beam is very low due to restriction of the mean frec path requirement, and then decreases as the square of the distance from the source [32]. Under the assumption of an isentropic, ideal gas expansion, however, the density in a supersonic jet is expected to be several orders of magnitude larger than that in an effusive beam, then falling off as the $2 / \gamma$ power of the distance downstream from the orifice $[32,37,38]$. In addition, the velocity for the effusive beam is governed by the Maxwell-Boltzmann distribution and characterized by the reservoir temperature [32]. The collisions occur as the particles with fast speed in the stream exceed the slow flow. In contrast, the jet beam velocity reaches its terminal value quickly in the part of expansion and cannot be speeded up any more, independently of the reservoir pressure or the distance downstream $[37,38]$. Increasing the distance downstream or the reservoir pressure may lead to a lower beam temperature, thereby narrowing the spread in the velocity distribution of the terminal mean velocity. It is interesting to note that the terminal mean velocity for a monatomic ideal gas corresponds to a factor of $(5 / 3)^{1 / 2}$ times as large as the root-mean-square velocity in an effusive beam [38]. The collision frequency per molecule occurring in an effusive beam, with the density $n$, collision cross section $\sigma$ and the mean velocity $v$ of molecules in the source, is estimated to be $2^{1 / 2} n \sigma v\left(7-4 \times 2^{1 / 2}\right) / 4$, explicitly independent of the temperature [32]. However, the collision frequency of molecules in a supersonic jet with the mean velocity $v_{0}$ and the temperature $T_{0}$ in the reservoir is determined by the formula $2^{1 / 2}$ ${ }^{\times} n \sigma v_{0}\left(T / T_{0}\right)^{1 / 2}$, depending on the square root of the beam temperature $T$ [32]. It is this factor that offsets the effect of the density, so that the molecules entrained in the jet beam can be considered free of collisions under the appropriate conditions. Finally, the translational temperature of molecules in the jet can be effectively governed depending upon the ratio of $X / D$ and the reservoir pressure. In this sense, the temperature (or kinetic energy) dependence measurement of orbital alignment effects will become accessible using a single supersonic jet, although it is beyond the scope of this work.

We have also attempted to operate the $\mathrm{Ca}$ oven as an effusive beam at the nozzle temperature $800 \pm 2$ $\mathrm{K}$ without introduction of the carrier gas Ar. The 
background pressure of $\mathrm{Ar}$ in the chamber was kept at about 100 mTorr. In this case, we have found that the fluorescence intensity becomes independent of the alignment of the polarized radiation; i.e. the polarization is completely depleted. The observation may be mainly attributed to the fact that the direction of the relative velocity between $\mathrm{Ca}$ and $\mathrm{Ar}$ differs in each collision, such that an individual $\Sigma$ or $\Pi$ symmetry curve cannot be effectively prepared and followed. The resulting orbital scrambling washes out the polarization.

Finally, we compare the current method to the crossed-beam configuration:

(1) The direction of the relative velocity vector in the device of supersonic jet is always along the molecular flow direction of the free jet, and is independent of the colliding species. The seeded $\mathrm{Ca}$ velocity is parallel to the carrier gas Ar velocity. The collisions here can be considered to be mainly along one dimension. In contrast, in the crossed-beam apparatus, the direction of the relative velocity vector varies depending upon the masses of the collision partners and their individual velocities [3,7]. The collision occurs in a two-dimensional plane, thus the relative velocity can spread over this plane. As a result, the direction of the polarized radiation in different systems must be adjusted to some extent for locking and following into a specific molecular state.

(2) Using the crossed-beam apparatus, the alignment effect is measured under single collision conditions. In our work, by appropriately selecting the operation conditions for the supersonic jet and the interaction region, the measured polarization can also result from a single-collision event. Under the condition of a very low background pressure (or extension to zero pressure), the resulting polarizations are found to be consistent with those in the crossed-beam experiments. However, the polarization can be gradually depleted as the background pressure increases because of the interference by quenching processes and the depolarization effect. This enables us to extract information on the role of the competing processes [ 341 . In addition, by varying the $X / D$ ratio and the reservoir pressure, it will be possible to study the temperature dependence of orbital alignment effects in energy transfer or chemical reactions.

(3) The optical alignment procedure for the current work is much easier than in a crossed-beam ex- periment, since only a two-beam interaction is involved instead of a three-beam alignment. In addition, in the crossed-beam configuration which employs a Ca effusive beam, a smaller number density at the interaction region is expected than that generated by a supersonic jet [32]. For instance, the $\mathrm{Ca}$ vapor at the nozzle temperature of $800 \mathrm{~K}$, having a reservoir density of $1.5 \times 10^{13}$ atoms $/ \mathrm{cm}^{3}$, can produce a number density $0.7 \times 10^{10}$ atoms $/ \mathrm{cm}^{3}$ at the interaction region in the crossed-beam experiment reported by Leone and co-workers [7]. In our case, we can expect the $\mathrm{Ca}$ density in the interaction region to be $4.7 \times 10^{10}$ atoms $/ \mathrm{cm}^{3}$, having about six times enhancement, using the same nozzle temperature. The above value is estimated according to the following equations $[32,37]$ :

$T / T_{0}=\left(\rho / \rho_{0}\right)^{\gamma-1}$

and

$T=T_{0}\left[1+\frac{1}{2}(\gamma-1) M_{\mathrm{eff}}^{2}\right]^{-1}$,

where $T_{0}$ and $\rho_{0}$ represent the temperature and number density at the reservoir; $T$ and $\rho$ are those at the interaction region. Comparing the alignment result shown in fig. 3 with that reported previously for the case of $\mathrm{Ca}-\mathrm{Ar}[7]$, we believe that a better signal-tonoise ratio obtained in this work is mainly attributed to this factor.

(4) The translational temperature of atoms in a supersonic jet is expected to be lower, thereby leading to a much narrower spread for the $\mathrm{Ca}$ velocity distribution. In this manner, the orbital scrambling process can be reduced, when compared to the use of a $\mathrm{Ca}$ effusive beam constructed in the crossedbeam apparatus. In addition, it is very possible to form a van der Waals complex such as CaAr under the condition of very low temperature. If a laser is tuned to the CaAr molecular transition, the $\Pi$ or $\Sigma$ molecular states can be selectively excited. In such a case, the alignment is prepared in a shorter internuclear distance and the orbital scrambling process should be minimized. This has already been demonstrated in the far wing scattering experiment $[21,22]$.

In summary, we have performed a supersonic jet experiment which allows one to study orbital alignment effects. The Ca $5{ }^{1} \mathrm{P}_{1}-5^{3} \mathrm{P}_{J}$ spin-changing collision by $\mathrm{Ar}$ is demonstrated. The observed polari- 
zation, $19 \% \pm 1.5 \%$ with a background pressure of about $55 \mathrm{mTorr}$, is quite consistent with that using the conventional crossed-beam configuration [7].

\section{Acknowledgement}

One of the authors (KCL) wishes to thank Professor Y.T. Lee and Professor W.H. Breckenridge for helpful discussions and for sending us preprints prior to publication. This work is financially supported by the National Science Council of the Republic of China under the contract 81-0417M-001-01.

\section{References}

[1] R.N. Zarc, Bcr. Bunsenges. Physik. Chem. 86 (1982) 422.

[2] C.T. Rettner and R.N. Zare, J. Chem. Phys. 77 (1982) 2416.

[3] S.R. Leone, in: Selectivity in chemical reactions, ed. J.C. Whitehead (Kluwer, Dordrecht, 1988) p. 245.

[4] L.J. Kovalenko, R.L. Robinson and S.R. Leone, J. Chem. Soc. Faraday Trans. II 85 (1989) 939.

[5] M.O. Hale, I.V. Hertel and S.R. Leone, Phys. Rev. Letters 53 (1984) 2296.

[6] I.V. Hertel, H. Schmidt, A. Bahring and E. Meyer, Rept. Progr. Phys. 48 (1985) 375.

[7] W. Bussert, D. Neuschafer and S.R. Leone, J. Chem. Phys. 87 ( 1987$) 3833$.

[8] B. Pouilly and M.H. Alexander, J. Chem. Phys. 86 (1987) 4790 .

[9] M.H. Alexander and B. Pouilly, J. Chem. Phys. 90 (1989) 5373;

B. Pouilly, J. Chem. Phys. 95 (1991) 5861.

[10] B. Pouilly, J.M. Robbe and M.H. Alexander, J. Chem. Phys. 91 ( 1989 ) 1658.

[11] A.Z. Devdariani and A.L. Zagrebin, Chem. Phys. Letters 131 (1986) 197.

[12] R.L. Robinson, L.J. Kovalenko, C.J. Smith and S.R. Leone, J. Chem. Phys. 92 (1990) 5260.

[13] A.G. Suits, H. Hou and Y.T. Lee, J. Phys. Chem. 94 (1990) 5672.

[14] H. Hulser, E.E.B. Campbell, R. Witte, H. Genger and I.V. Hertel, Phys. Rev. Letters 64 (1990) 392.
[15] A. Bahring, I.V. Hertel, E. Meyer, W. Meyer, N. Spies and H. Schmidt, J. Phys. B 17 (1984) 2859.

[16] M.X. Wang, M.S. de Vries and J. Weiner, Phys. Rev. A 33 (1986) 765 .

[17] E.W. Rothe, R. Theyunni, G.P. Reck and C.C. Tung, Phys. Rev. A 31 (1985) 1362.

[18] I.V. Hertel, H. Hofmann and K.A. Rost, Phys. Rev. Letters 38 ( 1977 ) 343.

[19] M.P.I. Manders, J.P.J. Driessen, H.C.W. Beijerinck and B.J. Verhaar, Phys. Rev. I etters 57 (1986) 1577.

[20] M.P.I. Manders, W.B.M. van Hoek, E.J.D. Vredenbregt, G.J. Sandker, H.C.W. Beijerinck and B.J. Verhaar, Phys. Rev. A 39 (1989) 4467.

[21] K.C. Lin, P.D. Kleiber, J.X. Wang, W.C. Stwalley and S.R. Leone, J. Chem. Phys. 89 (1988) 4771.

[22] S. Ananthamurthy, P.D. Kleiber, W.C. Stwalley and K.C. Lin, J. Chem. Phys. 90 (1989) 7605.

[23] C. Jouvet and B. Soep, Chem. Phys. Letters 96 (1983) 426.

[24] W.H. Breckenridge, C. Jouvet and B. Soep, J. Chem. Phys. 84 (1986) 1443.

[25] C. Jouvet, M.C. Duval, B. Soep, W.H. Breckenridge, C. Whitham and J.P. Visticot, J. Chem. Soc. Faraday Trans. II 85 (1989) 1133.

[26] W.H. Breckenridge, Accounts Chem. Kes. 22 (1989) 21.

[27] D.J. Funk and W.H. Breckenridge, J. Chem. Phys. 90 (1989) 2927.

[28] I. Wallace, J. Kaup and W.H. Breckenridge, J. Phys. Chem. 95 (1991) 8060.

[29] M.C. Duval, B. Soep and W.H. Breckenridge, J. Phys. Chem. 95 (1991) 7145 .

[30] K.C. Lin and C.T. Huang, J. Chem. Phys. 91 (1989) 5387.

[31] H.C. Chang, Y.L. Luo and K.C. Lin, J. Chem. Phys. 94 (1991) 3529.

[32] D.M. Lubman, C.T. Rettner and R.N. Zare, J. Phys. Chem. 86 (1982) 1129.

[33] T.A. Miller, Science 223 (1984) 545.

[34] Y.S. Duh and K.C. Lin, in preparation.

[35] W. Bussert and S.R. Leone, Chem. Phys. Letters 138 (1987) 269.

[36] C.H. Su, Y.S. Duh, K.C. Lin and W.T. Luh, in preparation.

[37] R.E. Smalley, L. Wharton and D.H. Levy, Accounts Chem. Res. 10 (1977) 139.

[38] D.R. Miller, in: Atomic and molecular beam methods, Vol. 1 , ed. G. Scoles (Oxford Univ. Press, Oxford, 1988) pp. $14-51$. 